\title{
Visible Light Communication Channel Models and Simulation of Coal Workface Energy Coupling
}

\author{
Yanrong Zhai ${ }^{1,2}$ and Shen Zhang ${ }^{1,2}$ \\ ${ }^{1}$ School of Information and Electrical Engineering, China University of Mining \& Technology, Xuzhou 221000, China \\ ${ }^{2}$ IOT Perception Mine Research Center, China University of Mining \& Technology, Xuzhou 221000, China \\ Correspondence should be addressed to Yanrong Zhai; zhaiyanrong@cumt.edu.cn
}

Received 24 July 2015; Revised 14 November 2015; Accepted 18 November 2015

Academic Editor: Hector E. Nistazakis

Copyright (c) 2015 Y. Zhai and S. Zhang. This is an open access article distributed under the Creative Commons Attribution License, which permits unrestricted use, distribution, and reproduction in any medium, provided the original work is properly cited.

\begin{abstract}
Due to their low energy consumption and small size, visible light communication systems have been widely used to eliminate communication-blind areas in coal workfaces. A workface visible light communication channel model and a mathematic model of energy coupling from the mining machine's workspace to its footpath were established to investigate the characteristics of optical signal transmission on the workface. This paper studies the effects of coal dust (double-layer particles encapsulated by moisture) on optical signal degradation. Simulation results revealed the presence of an optimized transmitter location, which maximized the coupled energy of the two space signals due to the blocking effect of metal columns.
\end{abstract}

\section{Introduction}

Fully mechanized coal faces are fundamentally different from other tunnel communication environments due to a continuously varying communication space caused by coal mining activities and electrostatic shielding impacts of hydraulic support metal columns on radio communication transmission [1]. The workface is surrounded by three metal faces and is supported by hydraulic supports; the cutter is a movable metallic machine. Hence, the skin effect on RF electromagnetic waves leads to a significant degradation of the wireless signals. These changing conditions make it difficult to use conventional radio communication systems in the workface. With visible light communication systems applied in the workface and LED as a base station (located on the roof of the hydraulic support), the location of the base station changes with the hydraulic support as the coal mining space varies, as shown in Figure 1. At a cost of simple LED modification, this method enables reduced workface space, lower power consumption, and improved safety.

To date, a variety of studies have been conducted on visible light communication system channel models. Chun et al.
[2] reported a simulation of signal-to-noise ratio of an organic LED applied in indoor illumination and communications. Ren et al. [3] improved the Barry recursive channel model by introducing optical source wiring topology $[4,5]$. Ding et al. [6] investigated the effects of LED array interference on the channel. Le and Jang [7] studied the distribution of visible light based on color channels. Lee et al. [8] proposed a channel model simulation in intelligent transportation. Sun and Akyildiz [9] treated mine tunnels as rectangles and established a waveguide channel model using ray-tracing methods. Gentile et al. [10] extended the waveguide channel model by considering the cross section and surface roughness. Also, ultrahigh frequency (UHF) simulcast signals and multipleinput multiple-output radio channel models were studied using ray-tracing methods [11, 12]. However, none of these reports presented a systematic study on the channel model's workface characteristics.

This paper consists of five sections: Section 2 establishes a workface channel model; Section 3 analyzes the effects of coal dust on optical signals; Section 4 presents the simulation and discussion; Section 5 contains the conclusion; the last section is the acknowledgement. 


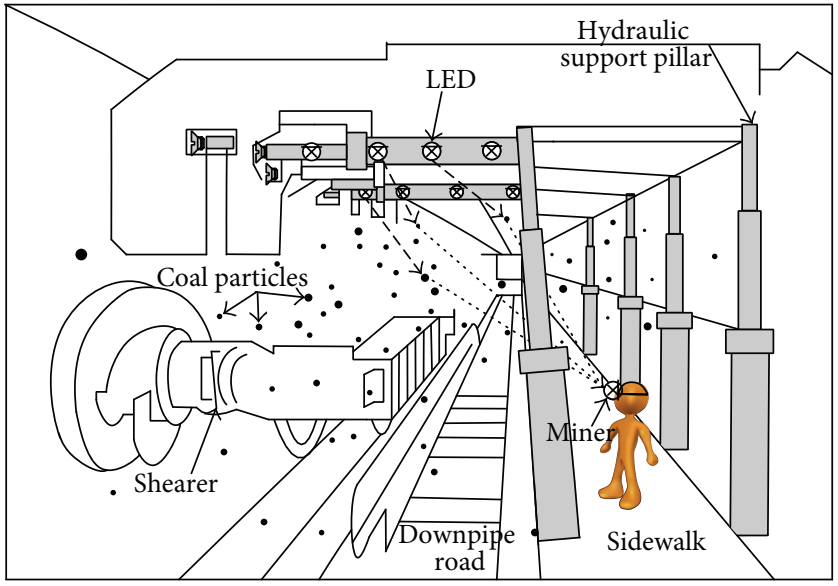

(a)

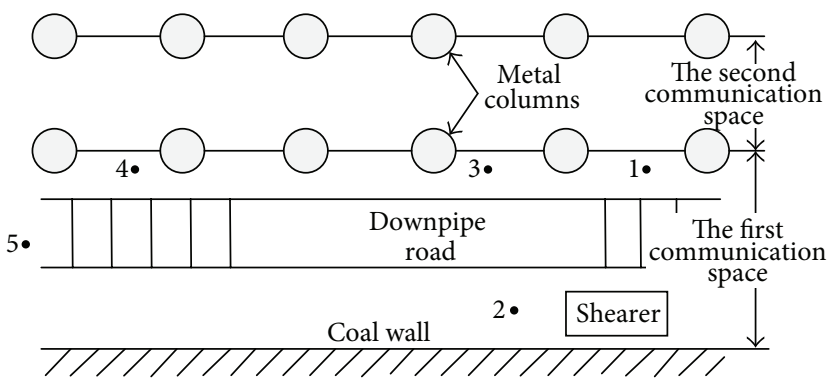

(b)

FIGURE 1: (a) Coal workface. (b) Coal workface schematic. 1: mining machine driver; 2: mining machine unloading coal; 3: moving frames; 4: places of multiple operation; 5: front slip.

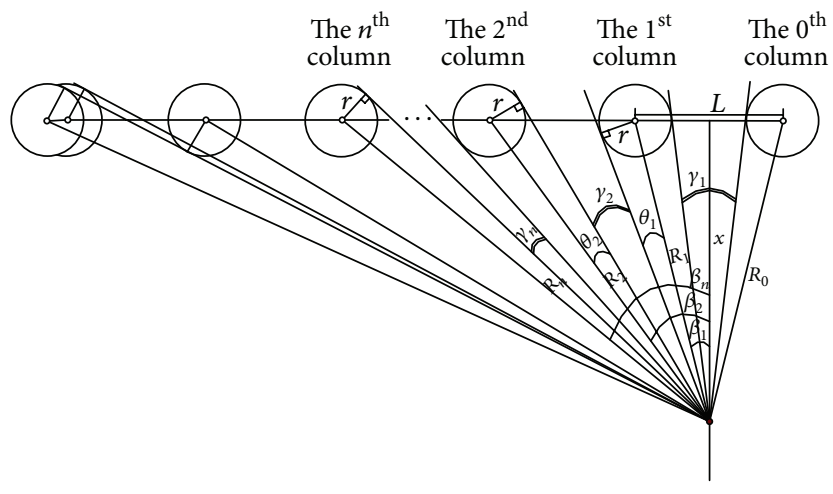

(a)

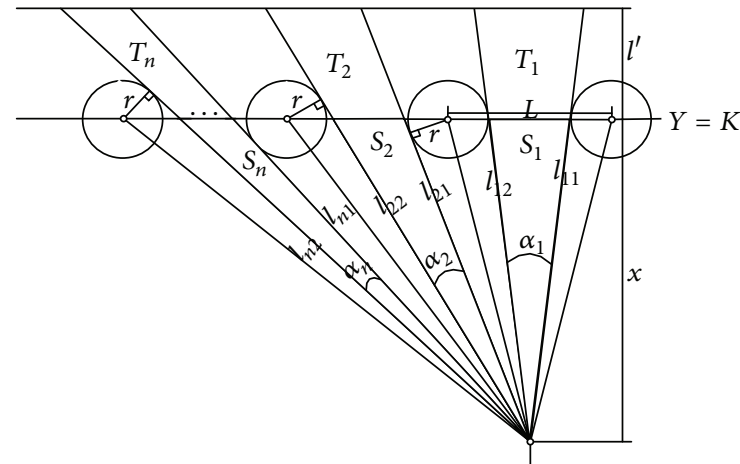

(b)

FIGURE 2: Calculation model for (a) coupling space angle and (b) coupling area and energy.

\section{Workface Channel Model}

The workface was divided into two individual communication spaces with hydraulic support columns as the boundary, as shown in Figure 2(b).

The signal energy couplings in the two communication spaces were investigated using ray transmission methods. Workface support exhibited two characteristics toward the radio wave transmission: the near-field indoor diffuse reflection model and far-field tunnel reflection model. Therefore, the signal transmission field can be divided into the near-field zone, the transition zone, and the far-field zone. In the nearfield region, the line-of-sight signal coupling and backward reflection signal coupling caused by workface support metal columns (forward reflection wave is back to the coal wall of first space) were observed. In the transition zone, partial backward reflection signal coupling was observed, although the energy level was negligible. The far-field zone can be regarded as a waveguide wall of the metal reflection wall.
2.1. Energy Coupling of Line-of-Sight Electromagnetic Waves in the Near-Field Zone. The distances between the midpoint of adjacent columns and their radii were $L$ and $r$, respectively. The transmitter was located in the median line of the 0 th and 1st column center, and the distance to the neutral point was $X$. The distance of the transmitter to the $n$th column center was $S_{n}$. The included angle between the straight line and tangent line of the launch point to the $n$th column center was $\theta_{n}$. The included angle between the straight line of the launch point to the $n$th column center and the median line with the 0 th and 1 st column center as an endpoint was $\beta_{n}$. The dashed area of the 2 nd communication space in the 1st communication was $S_{n}$. The area coupled to the 2 nd space was $T_{n}$ as shown in Figure 2(a).

The space of line-of-sight signal coupling in the $n$th and $(n-1)$ th column is defined as the coupling area $n$. The included angle $\gamma_{n}$ of the tangent line is defined as the coupling space angle $n$. The line linking the last column center with the signal coupling area and the launch point is defined as the 
coupling boundary. The energy coupling surface is at $y=K$. $P_{n 1}$ and $P_{n 2}$ are the critical coupling points of their respective coupling areas. The distance between the launch point and the coupling surface was $X$.

The coupling space angle can be calculated by

$$
\begin{aligned}
& \gamma_{1}=2\left(\beta_{1}-\theta_{1}\right) ; \\
& \gamma_{n}=\beta_{n}-\beta_{n-1}-\theta_{n}-\theta_{n-1}, \quad n \geq 2,
\end{aligned}
$$

where

$$
\begin{aligned}
& \beta_{n}=\arctan \left(\frac{L / 2+(n-1) \cdot L}{x}\right) ; \\
& \theta_{n}=\arcsin \left(\frac{r}{\sqrt{x^{2}+(L / 2+(n-1) \cdot L)^{2}}}\right) .
\end{aligned}
$$

The boundary column $n n$ of the near-field zone and transition zone satisfies the following conditions:

$$
\left(\beta_{n n}-\beta_{n n-1}\right) \leq\left(\theta_{n n}+\theta_{n n-1}\right)
$$

Coupling area can be calculated by

$$
\begin{aligned}
& T_{n}=S_{n} \cdot\left(\frac{\left(x+l^{\prime}\right)^{2}}{x^{2}}-1\right), \quad n \geq 1 ; \\
& S_{n}=\frac{x}{2}\left|P_{n 1}-P_{n 2}\right|, \quad n \geq 1,
\end{aligned}
$$

where

$$
\begin{aligned}
& P_{11}=\frac{-S_{1}}{x} ; \\
& P_{12}=\frac{S_{1}}{x} ; \\
& P_{n 1}=\frac{L}{2}+(n-2) \cdot L+\frac{r}{\sin \left(\arctan \left(x / l_{n 1}\right)\right)}, \quad n \geq 2 ; \\
& P_{n 2}=\frac{L}{2}+(n-1) \cdot L-\frac{r}{\sin \left(\arctan \left(x / l_{n 2}\right)\right)}, \quad n \geq 2 ; \\
& l_{11}=l_{12}=\frac{x}{\cos \left(\beta_{1}-\theta_{1}\right)} ; \\
& \beta_{1}=\arccos \left(\frac{x}{\sqrt{(L / 2)^{2}+x^{2}}}\right) ; \\
& \theta_{1}=\arcsin \left(\frac{r}{\sqrt{(L / 2)^{2}+x^{2}}}\right) ; \\
& l_{n 1}=\frac{\cos \left(\beta_{n}+\theta_{n}\right)}{n \geq 2 ;},
\end{aligned}
$$

$$
\begin{aligned}
& l_{n 2}=\frac{x}{\sin \left(\varepsilon_{n}+\theta_{n}\right)}, \quad n \geq 2 ; \\
& \varepsilon_{n}=\arcsin \left(\frac{x}{\sqrt{(L / 2+(n-1) \cdot L)^{2}+x^{2}}}\right) .
\end{aligned}
$$

Assuming that function $S$ was at a maximum value, the distance of the signal transmitter to the coupling surface was obtained. In this case, the area coupling to another line-ofsight signal space is maximized. At $y=K$, energy coupling to the $n$th space line-of-sight signal is

$$
E=\frac{(m+1)}{2 \pi d^{2}} \cos ^{m} \varphi \cos v
$$

where $m=\ln (1 / 2) / \ln \left(\cos \varphi_{1 / 2}\right)$ and $\varphi_{1 / 2}$ is half-power angle of LED. Consider

$$
I_{n}=\int_{z_{\min }}^{z_{\max }} \int_{P_{n 1}}^{P_{n 2}} E \frac{k}{\sqrt{x^{2}+k^{2}+z^{2}}} d x d z,
$$

where $z_{\max }$ and $z_{\min }$ are the maximum and minimum heights of the coupling surface, respectively. $P_{n 1}$ and $P_{n 2}$ are the critical points of the $n$th coupling space. The total energy current of coupling is

$$
I=\sum_{n=1}^{n n} I_{n}
$$

2.2. The Line-of-Sight Signals and Impulse Response of a Reflection Signal in the Near-Field Zone. Impulse response delays varied due to the different reflection paths. The impulse responses of line-of-sight signals and non-line-of-sight signal are [4]

$$
\begin{aligned}
& h^{(0)}(t ; S ; R) \approx \frac{n+1}{2 \pi} \cos ^{n}(\phi) d \Omega \operatorname{rect}\left(\frac{\theta}{\mathrm{FOV}}\right) \cdot \delta(t \\
& \left.-\frac{R}{c}\right) ; \\
& h^{(k)}(t ; S ; R) \approx \sum_{i=1}^{N} h^{(0)}\left(t ; s, \varepsilon_{i}\right) \otimes h^{(k-1)}\left(t ; s, \varepsilon_{i}\right)=\frac{n+1}{2 \pi} \\
& \quad \sum_{i=1}^{N} \frac{\rho_{i} \cos ^{n}(\phi) \cos (\theta)}{R^{2}} \\
& \quad \operatorname{rect}\left(\frac{2 \theta}{\pi}\right) \cdot h^{(k-1)}\left(t-\frac{R}{c} ;\{r, \widehat{n}, 1\}, R\right) \Delta A,
\end{aligned}
$$

where $A$ is the received explorer area and $\rho_{i}$ is the ray energy current reflection.

The receiving point, launching point, and reflection point were assumed to be $\left(x_{1}, y_{1}, z_{1}\right),\left(x_{2}, y_{2}, z_{2}\right)$, and $\left(x_{i}, y_{i}, z_{i}\right)$, respectively. As shown in Figure 3, four transmission paths were possible on a smooth reflection surface: the roof reflection ray, floor reflection ray, left column reflection ray, and right column reflection ray. 


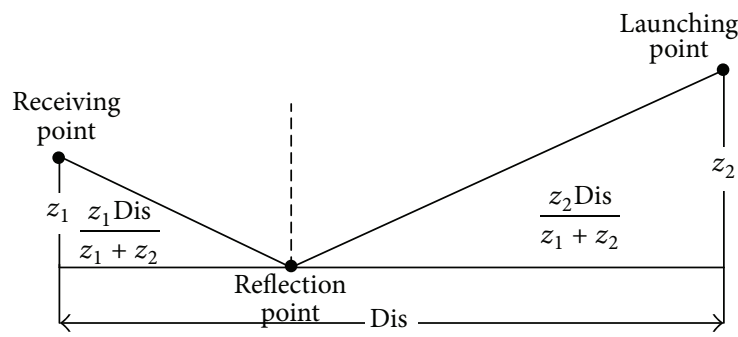

(a)

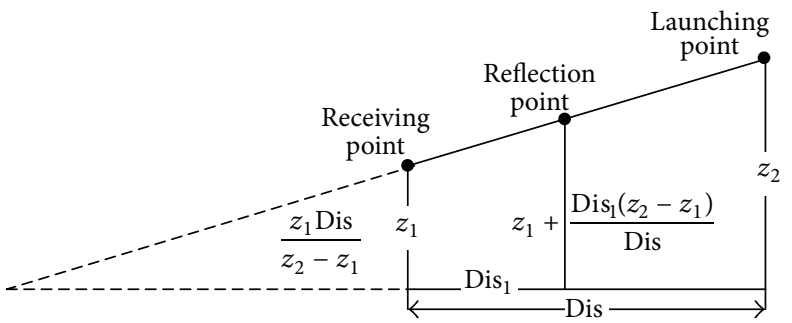

(b)

FIgURE 3: (a) Roof and floor reflection point coordinates; (b) metal column reflection point coordinates.

The roof or baseboard reflection point coordinates are

$$
\begin{gathered}
x_{1}-\frac{z_{1} \text { Dis }}{z_{1}+z_{2}} \cos a ; \\
\frac{z_{1} \text { Dis }}{z_{1}+z_{2}} \sin a,
\end{gathered}
$$

where $z$ is height of roof or baseboard:

$$
\begin{aligned}
\text { Dis } & =\sqrt{\left(x_{1}-x_{2}\right)^{2}+\left(y_{1}-y_{2}\right)^{2}} ; \\
\sin a & =\frac{\left|y_{1}-y_{2}\right|}{\text { Dis }} .
\end{aligned}
$$

The roof or baseboard reflection point coordinates in the $n$th coupling space metal column are

$$
\left(P_{n 1 / n 2}, k, z_{1}, \frac{\text { Dis }\left|z_{1}-z_{2}\right|}{\text { Dis }}\right) \text {. }
$$

\section{Attenuation Effects of Coal Dust Particles on Optical Signal}

Coal dust particles have strong absorption effects on electromagnetic and light waves. The working coal cutter generated a considerable amount of floating coal dust particles, resulting in light signal attenuation due to scattering and absorption by these suspending particles. The relation between incident light intensity and transmission light intensity can be expressed as follows [13]:

$$
I=I_{0} e^{\left(-(3 / 2) \cdot\left(C L K_{e} / d\right)\right)},
$$

where $C$ is the number of suspending particles in the unit volume $\left(/ \mathrm{cm}^{3}\right), L$ is light path $(\mathrm{cm})$, and $K_{e}$ is light attenuation coefficient: $K_{e}=Q_{\text {sca }}+Q_{\text {abs }}$.

Currently, the most widely used method for workface dust prevention is coal seam water infusion. This method causes dust fall and wetting; water vapor is produced and absorbed by coal particle surfaces. A previous study [14] reported that the contact angle of coal particles with an average grain size of $3.75-19.71 \mu \mathrm{m}$ ranged from $87.39^{\circ}$ to $90.00^{\circ}$, indicating that the particles were hydrophobic, as shown in Figure 5. Therefore, workface coal dust can be regarded as a condensation nucleus covered by a thin water vapor layer. Coal dust particles settle when they coagulate with sufficient water molecules.

Mie scattering is a rigorous solution to scattering problems of uniform spherical particles of any size. The scattering absorption effect of dust particles of diameters comparable to the radiation wavelength can be investigated using Mie scattering. The Mie factors of double-layer particles $\left(a_{n}\right.$ and $\left.b_{n}\right)$ are [15]

$$
\begin{aligned}
& a_{n}=\frac{\left(\widetilde{D}_{n} / m_{2}+n / y\right) \psi_{n}(y)-\psi_{(n-1)}(y)}{\left(\widetilde{D}_{n} / m_{2}+n / y\right) \xi_{n}(y)-\xi_{n-1}(y)} \\
& b_{n}=\frac{\left(m_{2} \widetilde{G}_{n}+n / y\right) \psi_{n}(y)-\psi_{n-1}(y)}{\left(m_{2} \widetilde{G}_{n}+n / y\right) \xi_{n}(y)-\xi_{n-1}(y)}
\end{aligned}
$$

where

$$
\begin{aligned}
& \widetilde{D}_{n}=\frac{D_{n}\left(m_{2} y\right)-A_{n} \chi_{n}^{\prime}\left(m_{2} y\right) / \psi_{n}\left(m_{2} y\right)}{1-A_{n} \chi_{n}\left(m_{2} y\right) / \psi_{n}\left(m_{2} y\right)} \\
& \widetilde{G}_{n}=\frac{D_{n}\left(m_{2} y\right)-B_{n} \chi_{n}^{\prime}\left(m_{2} y\right) / \psi_{n}\left(m_{2} y\right)}{1-B_{n} \chi_{n}\left(m_{2} y\right) / \psi_{n}\left(m_{2} y\right)} \\
& A_{n}=\psi_{n}\left(m_{2} x\right) \frac{m_{n}\left(m_{1} x\right)-D_{n}\left(m_{2} x\right)}{m D_{n}\left(m_{1} x\right) \chi_{n}\left(m_{2} x\right)-\chi_{n}^{\prime}\left(m_{2} x\right)} \\
& B_{n}=\psi_{n}\left(m_{2} x\right) \frac{D_{n}\left(m_{1} x\right) / m-D_{n}\left(m_{2} x\right)}{D_{n}\left(m_{1} x\right) \chi_{n}\left(m_{2} x\right) / m-\chi_{n}^{\prime}\left(m_{2} x\right)} \\
& D_{n}(z)=\frac{\psi_{n}^{\prime}(z)}{\psi_{n}(z)} ; \\
& m=\frac{m_{2}}{m_{1}} ; \\
& \chi_{n}(z)=-z \sqrt{\frac{\pi}{2 z}} Y_{n+0.5}(z) ; \\
& \psi_{n}(z)=z \sqrt{\frac{\pi}{2 z}} J_{n+0.5}(z) ; \\
& \xi_{n}=z\left(\sqrt{\frac{\pi}{2 z}} J_{n+0.5}(z)+i \sqrt{\frac{\pi}{2 z}} Y_{n+0.5}(z)\right)
\end{aligned}
$$

$J$ and $Y$ are Bessel functions of the first kind order $n$ and the second kind order $n$, respectively. 
The corresponding extinction efficiency factor $Q_{\text {est }}$, forward scattering factor $Q_{\text {sca }}$, and absorption factor $Q_{a b s}[16]$ are expressed as

$$
\begin{aligned}
& Q_{\text {sca }}=\frac{2}{x^{2}} \sum_{n=1}^{n_{c}}(2 n+1)\left(\left|a_{n}\right|^{2}+\left|b_{n}\right|^{2}\right) ; \\
& Q_{\text {est }}=\frac{2}{x^{2}} \sum_{n=1}^{n_{c}}(2 n+1) \Re\left\{a_{n}+b_{n}\right\} ; \\
& Q_{\mathrm{abs}} \approx Q_{\text {est }}-Q_{\text {sca }} .
\end{aligned}
$$

The backward-scattering coefficient $Q_{b a c}$ and the average cosine of the scattering angle with respect to power are as follows [17]:

$$
\begin{aligned}
& Q_{\mathrm{bac}}=\frac{1}{x^{2}}\left|\sum_{n=1}^{n_{c}}(2 n+1)(-1)^{n}\left(a_{n}-b_{n}\right)\right|^{2} ; \\
& Q_{\mathrm{sca}}\langle\cos \theta\rangle=\frac{4}{x^{2}}\left\{\sum_{n=1}^{\infty} \frac{n(n+2)}{n+1} \operatorname{Re}\left(a_{n} a_{n+1}^{*}+b_{n} b_{n+1}^{*}\right)\right. \\
& \left.\quad+\sum_{n+1}^{\infty} \frac{2 n+1}{n(n+1)} \operatorname{Re}\left(a_{n} b_{n}^{*}\right)\right\} ; \\
& n_{c}=x+4 x^{1 / 3}+1,
\end{aligned}
$$

where Re stands for the real part.

\section{Simulation and Analysis}

4.1. The Effect of Coal Dust Water Vapor on the Light Signal. Simulations based on grain size of $2 \mu \mathrm{m}, 4 \mu \mathrm{m}, 7 \mu \mathrm{m}$, and $10 \mu \mathrm{m}$ using Mie coefficients of different water vapor layer thicknesses were conducted and shown in Figure 4.

According to Figure 4, the scattering absorption coefficient fluctuated with water vapor thickness and the fluctuation degree increased with the thickness of the water vapor layer. The coefficients tend to be stable and eventually converged to a straight line as thickness decreased. Additionally, the effect of water vapor layer thickness on the extinction scattering coefficient increased as the nuclei radius decreased, indicating that the water vapor covering coal dust has an effect on coal dust's light scattering absorption. Therefore, coal dust characteristics and water vapor layer thickness must be investigated to understand the effects of coal dust particles on light signals.

Dong et al. [14] used a German automatic measuring instrument (operating manual DACT21) to perform QLM-90 airstream grinding on coal samples obtained from five coal preparation plants (QTH, TF, DD, XS, and QX). The elemental composition and ash content of coal in different mining areas have been described elsewhere [14]. Based on different turbo classifying convolution, coal samples from each plant were divided into 4 groups with different average grain sizes. The water/coal wetting balance contact angles of the 20
TABLE 1: Characteristics in simulation for signal pulse response and energy coupling.

\begin{tabular}{lc}
\hline $\begin{array}{l}\text { Total grain swarm average grain size } \\
\text { (weighted average) }\end{array}$ & $7.6018 \mu \mathrm{m}$ \\
\hline Water vapor thickness & $2.6596 \mu \mathrm{m}$ \\
\hline Extinction coefficient & 2.1162 \\
\hline Scattering coefficient & 1.4115 \\
\hline Absorption coefficient & 0.7011 \\
\hline
\end{tabular}

coal sample groups were measured; the results are shown in Figure 5.

According to Figure 5, the contact angles of four coal samples groups with grain sizes of $3-20 \mu \mathrm{m}$ from QTH, $\mathrm{DD}, \mathrm{XS}$, and QX remained constant at $89.9^{\circ}$. The coal sample contact angle from TF was slightly smaller than other samples with the same grain size; the fluctuation range was about $1.5^{\circ}$.

According to the length measuring method of the wetting contact angle, the water vapor layer thickness absorbed around coal dust particles was estimated by $\sin \theta=2 \mathrm{hr} /\left(\mathrm{h}^{2}+\right.$ $\left.r^{2}\right)$ or $\sin (\theta / 2)=h / r$. The corresponding scattering absorption coefficients were obtained and shown in Figure 6.

According to Figure 6, the Mie coefficients of DD and TF samples with a $7.6 \mu \mathrm{m}$ grain size (except for the back scattering coefficient) were almost the same, demonstrating that the differences in the contact angle did not affect the Mie coefficients. Therefore, the scattering absorption coefficient of wet coal dust particles (3-20 $\mu \mathrm{m})$ was determined by the grain radius.

Since workface dust consists of particles with different grain sizes, the average granularity calculated by statistical mathematics was used for simulations. Zuo [18] investigated the statistics of particles from Bulianta Mine of Shendong Coal Group Corporation Limited 22306 comprehensive mining workface coal cutter driving position, coal cutter coal falling position, moving frame position, and multiple processes position by using a Winner 99 Microscopic Particle Image Analyzer as shown in Figure 1(b). Set the coal dust grain size from these five positions as grain swarm; the particle characteristics in the simulations for the signal pulse response and energy coupling were obtained and shown in Table 1.

4.2. Energy Coupling of Line-of-Sight Signals. According to the parameters of the ZY9200/25/50D hydraulic support, the central distance was $1.75 \mathrm{~m}$, the supporting height was $3 \mathrm{~m}$, the supporting width was $1.76 \mathrm{~m}$, the cylinder diameter was $400 \mathrm{~mm}$, and the pillar diameter was $380 \mathrm{~mm}$. A MLS3-170 coal cutter and DGWD-180 conveyor were used. The matched size was $1972 \mathrm{~mm}$, the top beam length was $3700 \mathrm{~mm}$, and the line-of-sight signals and first-order reflection NLOS signal channel were simulated. The emitting and receiving point coordinates were $(10,6,2.5)$ and $(10,1,0.8)$, respectively. The LED half power angle was $60^{\circ}$. 


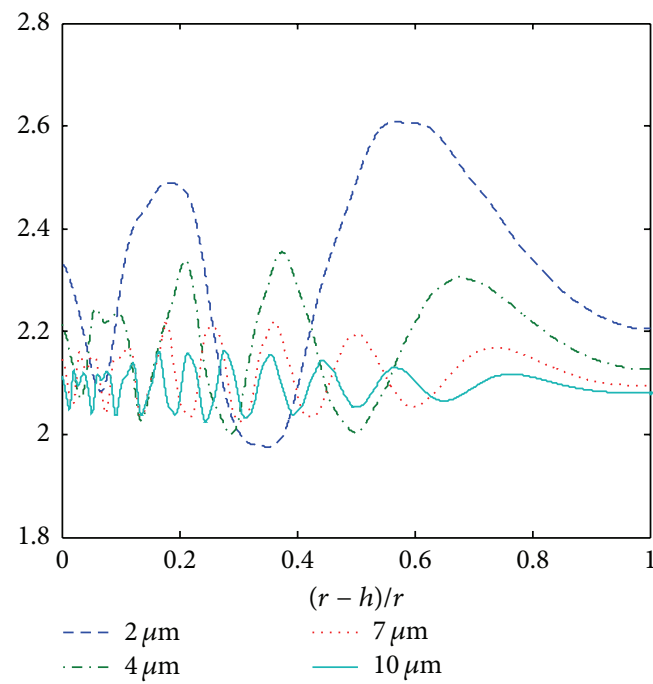

(a) $Q_{\text {est }}$

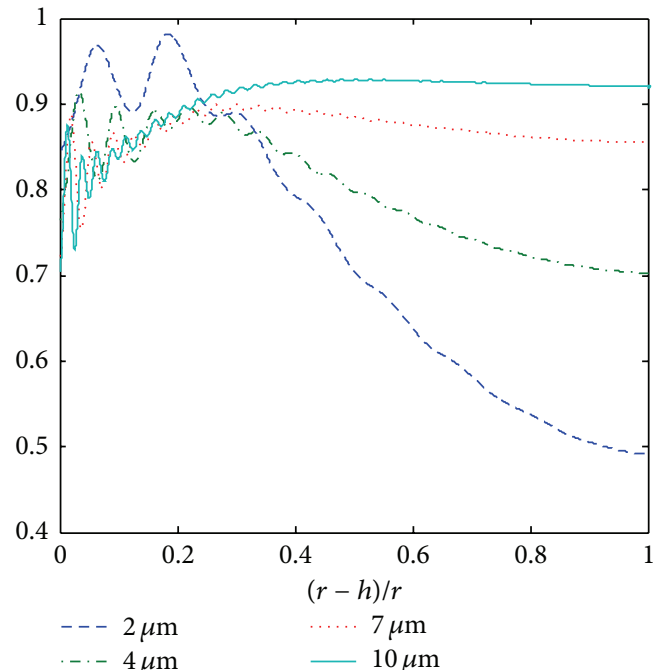

(c) $Q_{a b s}$

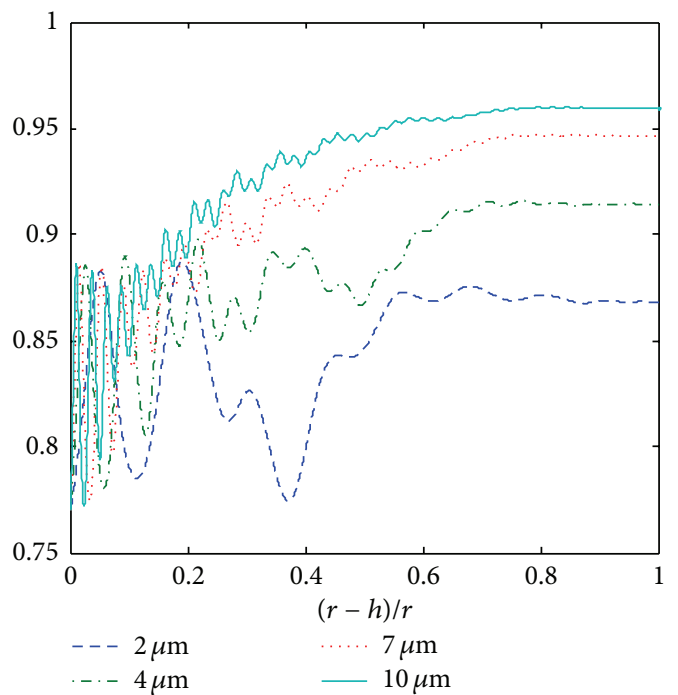

(e) $Q_{\text {sca }}\langle\cos \theta\rangle$

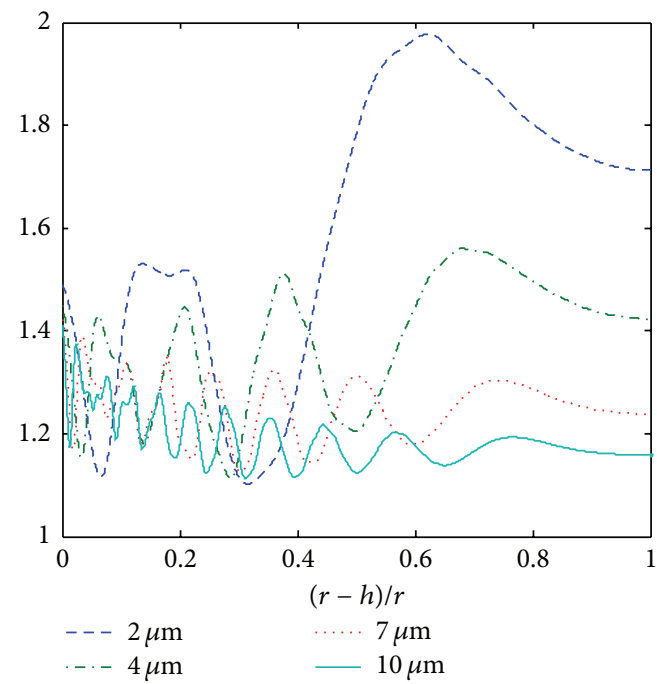

(b) $Q_{\text {sca }}$

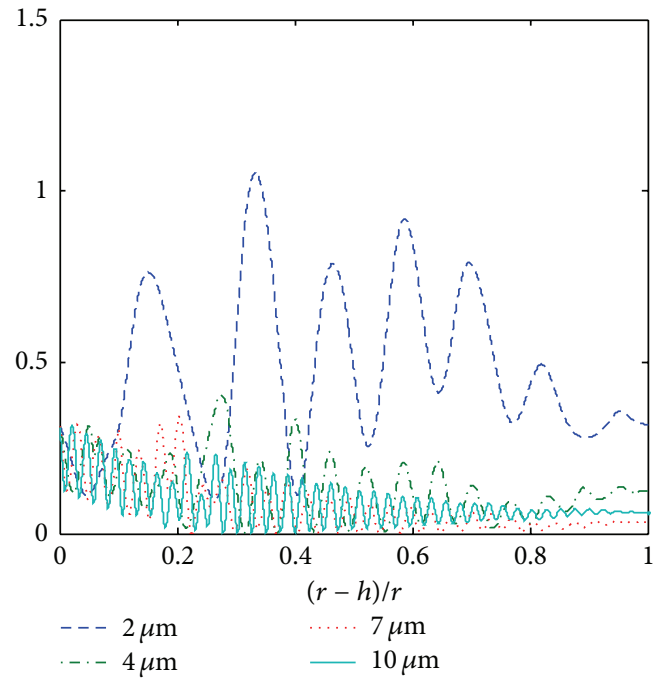

(d) $Q_{b a c}$

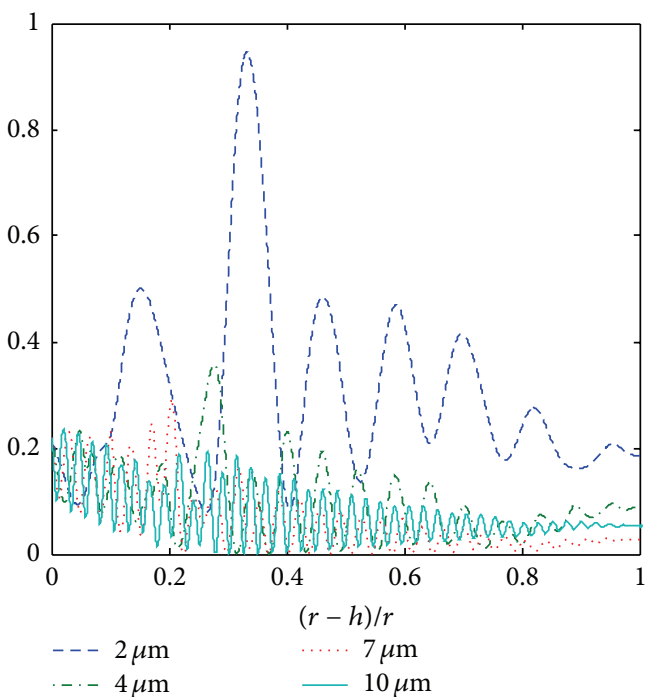

(f) $Q_{\text {bac }} / Q_{\text {sca }}$

FIGURE 4: Scattering absorption coefficients of particles with different grain sizes versus water vapor layer thickness. 


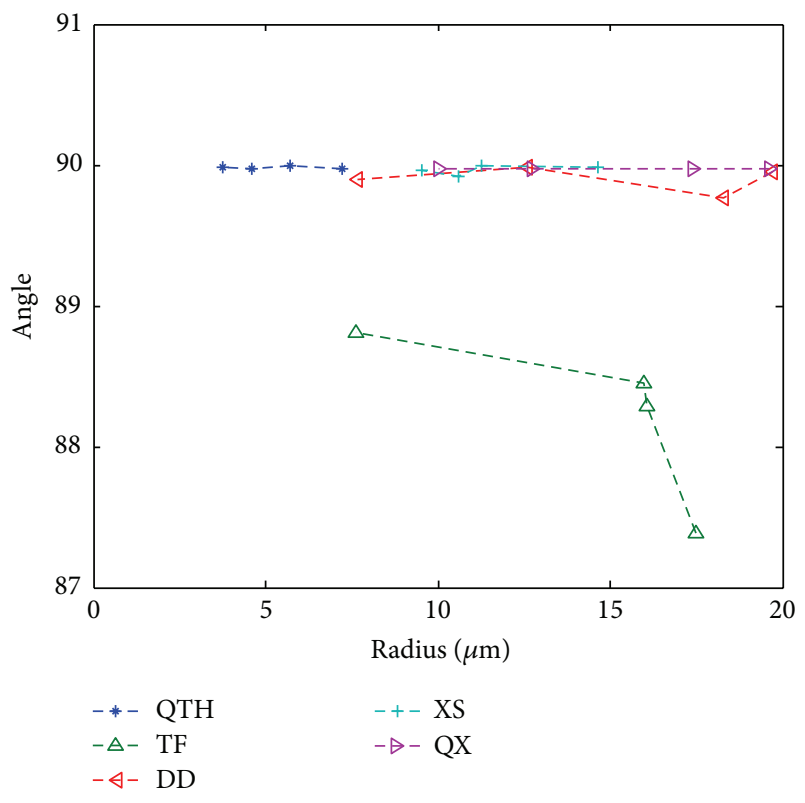

FIGURE 5: Contact angle of different coal samples.

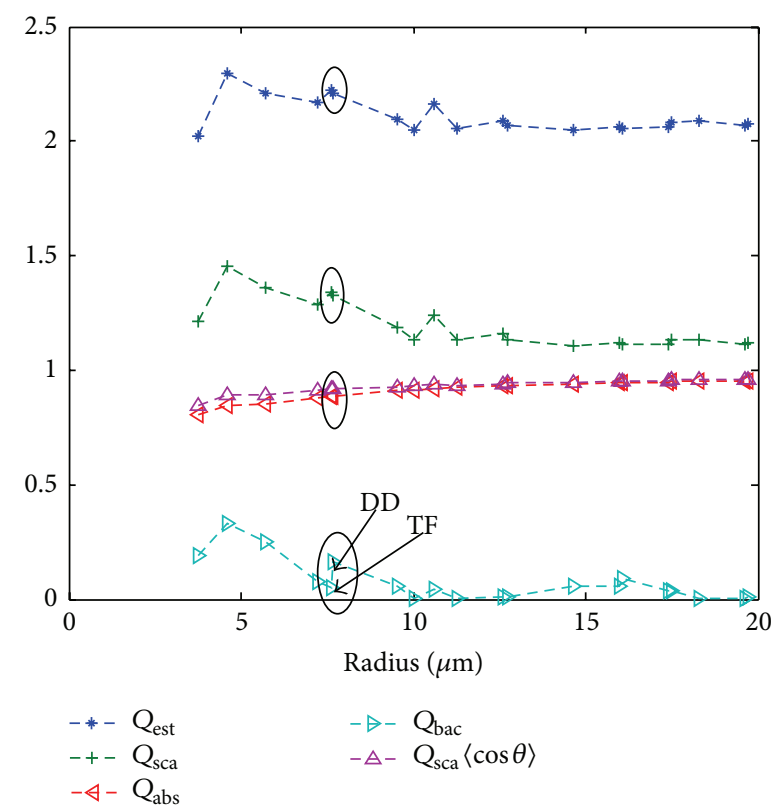

FIGURE 6: Relation between the contact angle and the scattering absorption coefficient.

Figure 7 shows an illustration of the light energy stream reflection from air to iron. The reflecting ratio remained constant at 0.56 if the reflecting angle was between 0 and $60^{\circ}$. Figure 8 shows the pulse response of line-of-sight signals and first-order NLOS reflection signals. Time delays caused by the direct path signal and nearest pillar reflection signal were comparable. However, signal strength decreased as the pillar distance increased. The intensity of forward path signals

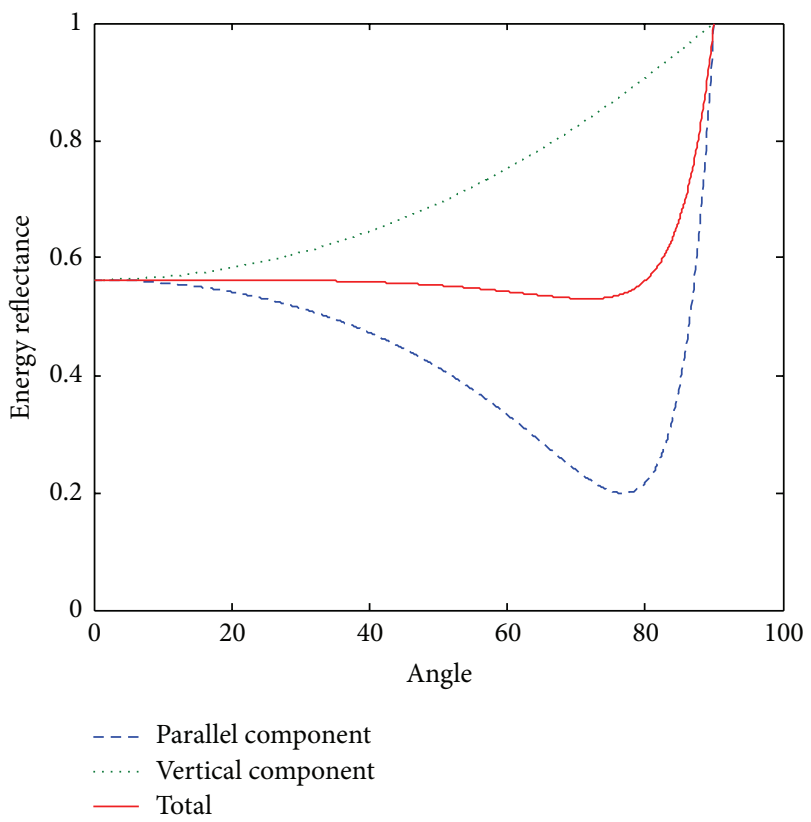

FIGURE 7: Energy reflection coefficient of $650 \mathrm{~nm}$ light from air to iron.

(Figure 8(a)) was higher than that of reflection path signals. As shown in Figure 8(b), only one peak of reflection path signal intensity was observed between the first pillar and the second pillar, which can be attributed to the signal overlapping. Additionally, the signal intensity via the reflection path of the roof (Figure $8(\mathrm{c})$ ) was significantly higher than that via the reflection path of the floor (Figure $8(d)$ ) as the reflection medium on the floor was coal while the reflection medium on the roof was steel.

According to Figures 9(a) and 9(b), trends of the coupling angle and each coupling area were highly similar. However, the trends of the coupling angle and coupling area in the first space were different from those in other spaces. At short distances, coupling angles, coupling areas, and coupling energy in other spaces were negligible. This can be attributed to the fact that the coupling angle and coupling area were inversely proportional to the distance, as the light source was along the perpendicular bisector of the first space. At short distances, the light source was roughly parallel to the coupling boundary, and the coupling angle was negligible. As the distance increased, energy coupling was observed in different coupling spaces. Figure 9(c) shows that when the emitting point was $1.3 \mathrm{~m}$ away from the coupling plan, the total coupling energy stream was maximized. In Figure 9(d), since the electromagnetic wave attenuated faster in the visible light waveband, the light energy attenuation scales in different areas were different. Nevertheless, as distance increased, this scale tended to be stable at 0.1 , and a coupling energy peak point existed when the emitting point was coupled to every field. Although the coupling area of first field area increased as the distance decreased, when the emitter was close to the coupling plan, the existing line-of-sight signal coupling area 


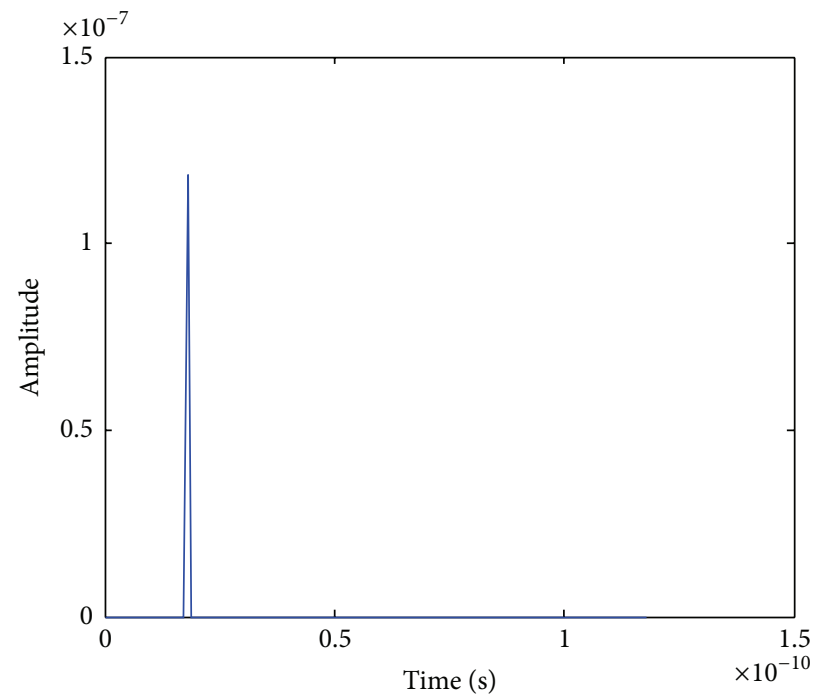

(a) Line-of-sight

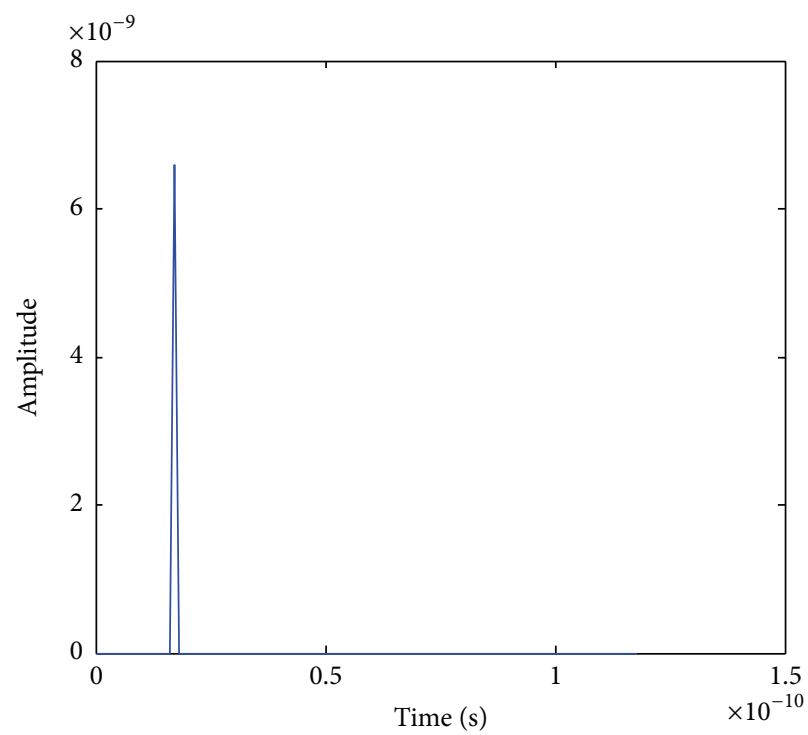

(c) The roof reflection ray

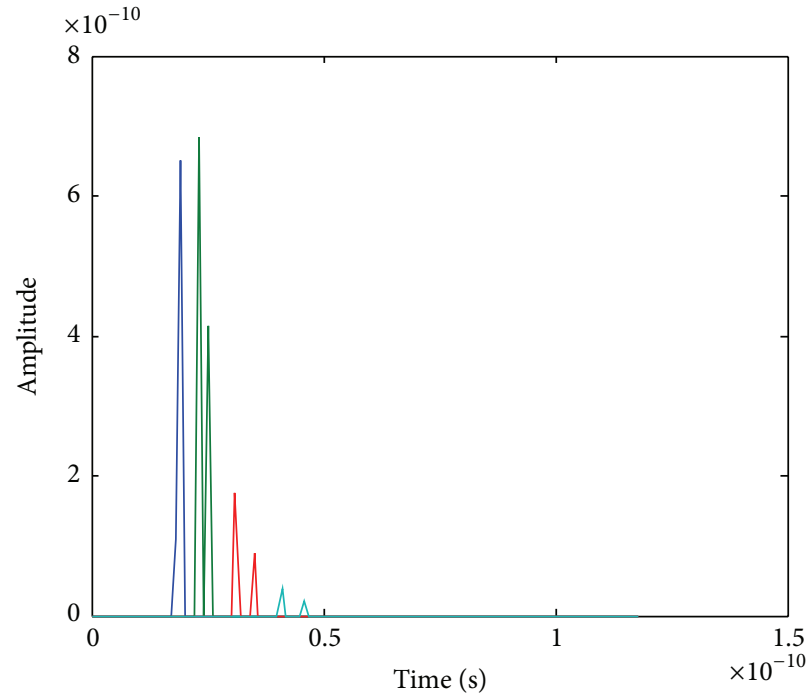

(b) The left and right columns reflection rays

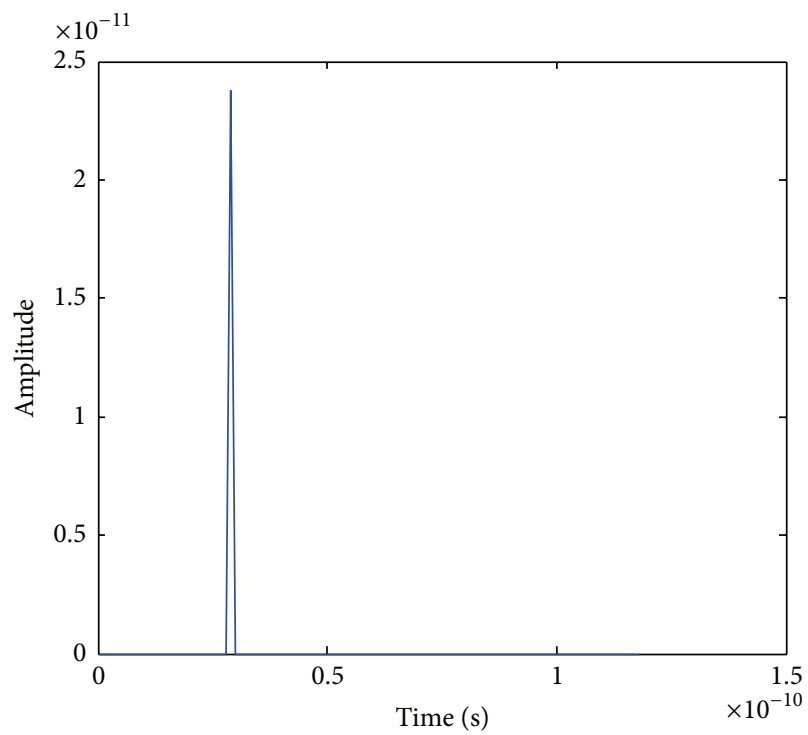

(d) The floor reflection ray

FIGURE 8: Line-of-sight and first-order reflecting signal pulse response.

decreased due to the pillar sheltering effect; thus, a peak value still existed in the first field area. Relative to the distance from the emitter and coupling plan, the pillar radius was so small that it can be taken as a point, and no energy coupling peak value was observed in the first coupling field area.

\section{Conclusions}

Due to the presence of water vapor, coal dust particles should be regarded as water vapor-covered double-layer particles for the study of attenuation effects caused by coal dust particles on wireless light signals. Also, the slight change of the coal dust wetting contact angle did not have a significant effect on the grain scattering absorption coefficient. Therefore, the water vapor thickness of coal dust particles in the workface was determined by the grain size. For signal couplings in different communication spaces, a maximum energy coupling value was presented in every field due to the sheltering effect of the metal pillar. This benefits the determination of emitter position and receiver signal energy and power analysis.

\section{Conflict of Interests}

The authors declare that there is no conflict of interests regarding the publication of this paper. 


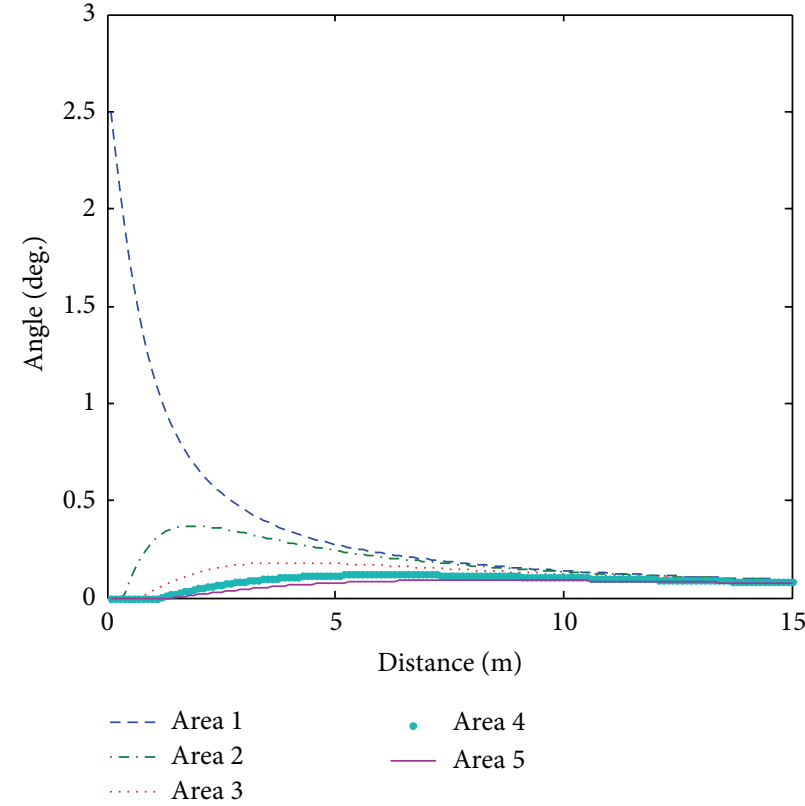

(a) Coupling angle

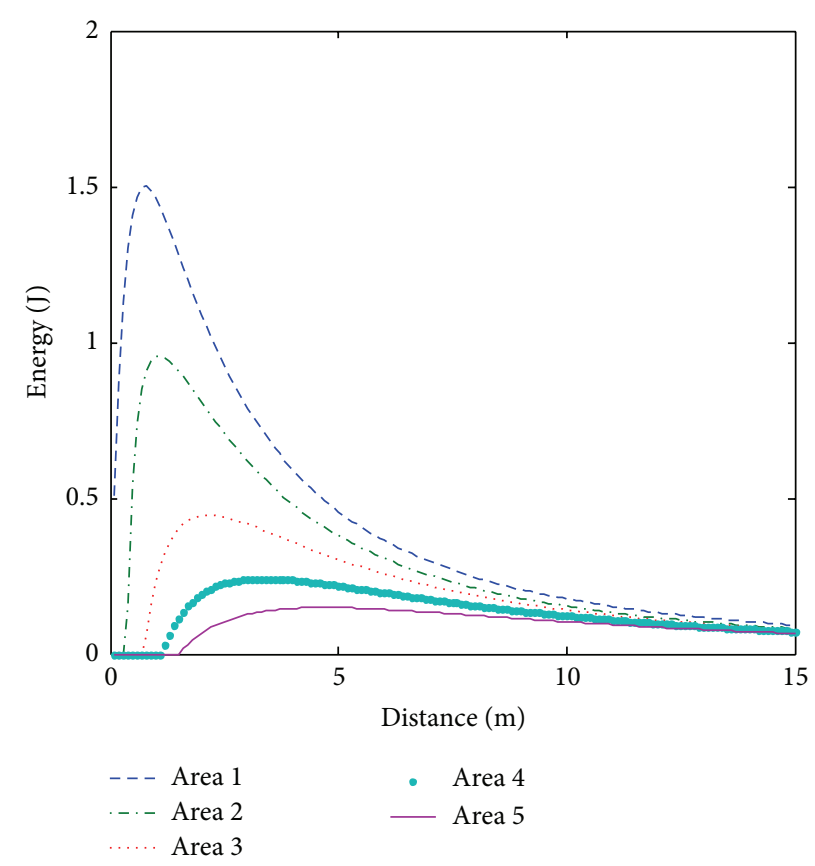

(c) Coupling energy

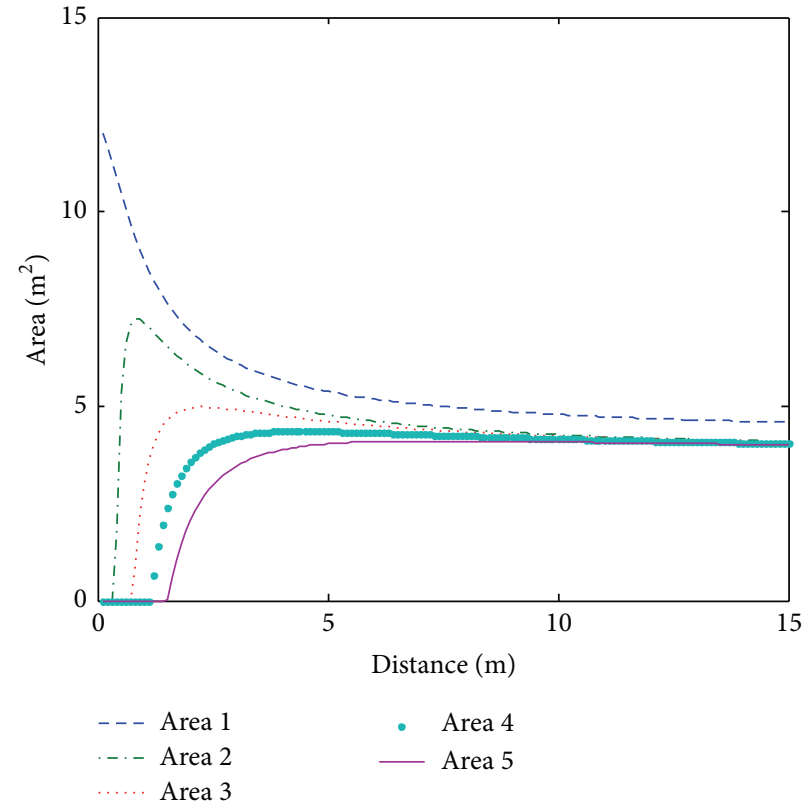

(b) Coupling area

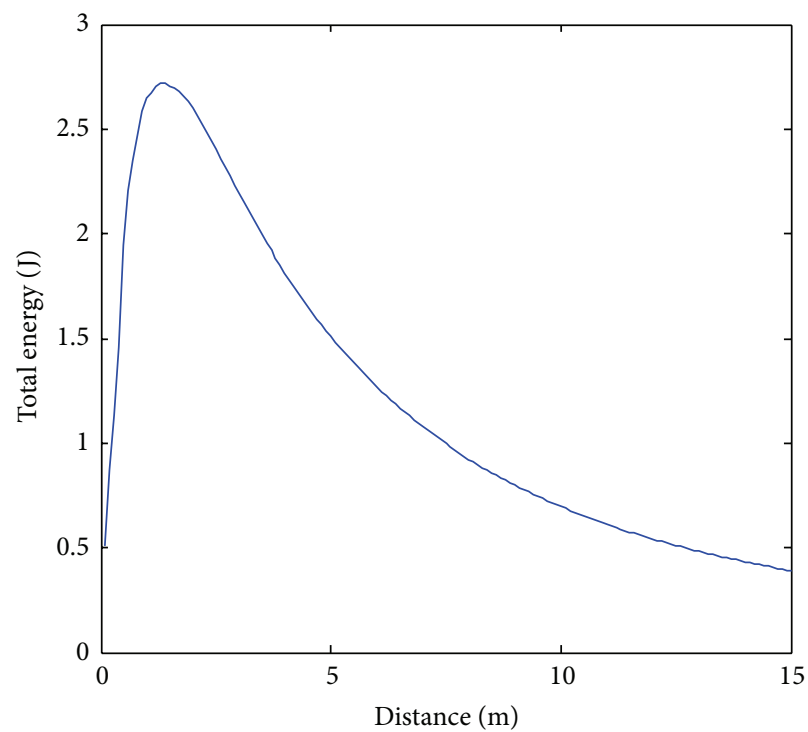

(d) Coupling total energy

FIGURE 9: Simulation of coupling field area coupling space and coupling energy.

\section{Acknowledgments}

The authors gratefully acknowledge the support of The National Science \& Technology Pillar Program of China (2013BAK06B05) and Postgraduate Training Innovation Project of Jiangsu Province (KYLX_1387).

\section{References}

[1] X. L. Feng and J. S. Qian, "Architecture of wireless multimedia mesh network nodes for longwall coal mine automation," in Proceedings of the 3 rd International Conference on Information and Computing (ICIC '10), vol. 2, pp. 42-45, IEEE, Wuxi, China, June 2010. 
[2] H. Chun, C.-J. Chiang, and D. C. O’Brien, "Visible light communication using OLEDs: illumination and channel modeling," in Proceedings of the International Workshop on Optical Wireless Communications (IWOW '12), pp. 1-3, IEEE, Pisa, Italy, October 2012.

[3] G. H. Ren, S. Y. He, and Y. L. Yang, "An improved recursive channel model for indoor visible light communication systems," Information Technology Journal, vol. 12, no. 6, pp. 1245-1250, 2013.

[4] J. R. Barry, J. M. Kahn, W. J. Krause, E. A. Lee, and D. G. Messerchmitt, "Simulation of multipath impulse response for indoor wireless optical channels," IEEE Journal on Selected Areas in Communications, vol. 11, no. 3, pp. 367-379, 1993.

[5] K. Lee, H. Park, and J. R. Barry, "Indoor channel characteristics for visible light communications," IEEE Communications Letters, vol. 15, no. 2, pp. 217-219, 2011.

[6] J. P. Ding, K. Wang, and Z. G. Xu, "Impact of LED array simplification on indoor visible light communication channel modeling," in Proceedings of the 9th International Symposium on Communication Systems, Networks \& Digital Signal Processing (CSNDS '14), pp. 1159-1164, IEEE, Manchester, UK, July 2014.

[7] N.-T. Le and Y. M. Jang, "Smart color channel allocation for visible light communication cell ID," Optical Switching and Networking, vol. 15, pp. 75-86, 2015.

[8] S. J. Lee, J. K. Kwon, S. Y. Jung, and Y. H. Kwon, "Simulation modeling of visible light communication channel for automotive applications," in Proceedings of the 2012 15th International IEEE Conference on Intelligent Transportation Systems, pp. 463468, Anchorage, Alaska, USA, September 2012.

[9] Z. Sun and I. F. Akyildiz, "Channel modeling and analysis for wireless networks in underground mines and road tunnels," IEEE Transactions on Communications, vol. 58, no. 6, pp. 17581768, 2010.

[10] C. Gentile, F. Valoit, and N. Moayeri, "A raytracing model for wireless propagation in tunnels with varying cross section," in Proceedings of the IEEE Global Communications Conference (GLOBECOM '12), pp. 5027-5032, IEEE, Anaheim, Calif, USA, December 2012.

[11] Y. P. Zhang, Y. Hwang, and J. H. Sheng, "Propagation characteristics of UHF simulcast signals in tunnel environments," in Proceedings of the International Conference on Communication Technology (ICCT'96), vol. 1, pp. 457-460, IEEE, Beijing, China, May 1996.

[12] S.-H. Yao and X.-L. Wu, "Modeling for MIMO wireless channels in mine tunnels," in Proceedings of the International Conference on Electric Information and Control Engineering (ICEICE '11), pp. 520-523, IEEE, Wuhan, China, April 2011.

[13] L. Wang, Research on Retrieval Algorithm of Particle Size Distribution Based on Spectral Extinction Method, Harbin Institute of Technology, 2013.

[14] P. Dong, Z. J. Shan, and Z. Li, "Study on the surface wet characteristic of ultrafine coal powder," Journal of China Coal Society, vol. 29, no. 3, pp. 346-349, 2004.

[15] N. G. Volkov and V. Y. Kovach, "Scattering of light by inhomogeneous spherically symmetrical aerosol particles," Izvestiya Atmospheric and Oceanic Physics, vol. 26, no. 5, pp. 381-385, 1990.

[16] H. C. V. Hulst, Light Scattering by Small Particle, Wiley, New York, NY, USA, 1957.
[17] W. E. Meador and W. R. Weaver, "Two-stream approximations to radiative transfer in planetary atmospheres: a unified description of existing methods and a new improvement," Journal of the Atmospheric Sciences, vol. 37, no. 3, pp. 630-643, 1980.

[18] Q. M. Zuo, Diffusion Law and Dust Control Techniques of Large Mining Height Fully-Mechanized Face, China University of Mining and Technology, Xuzhou, China, 2014. 


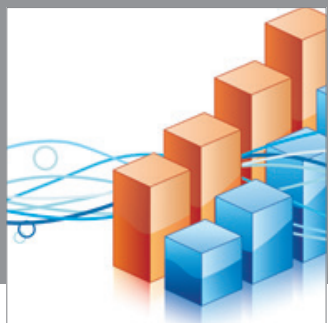

Advances in

Operations Research

mansans

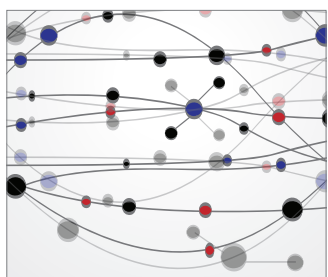

The Scientific World Journal
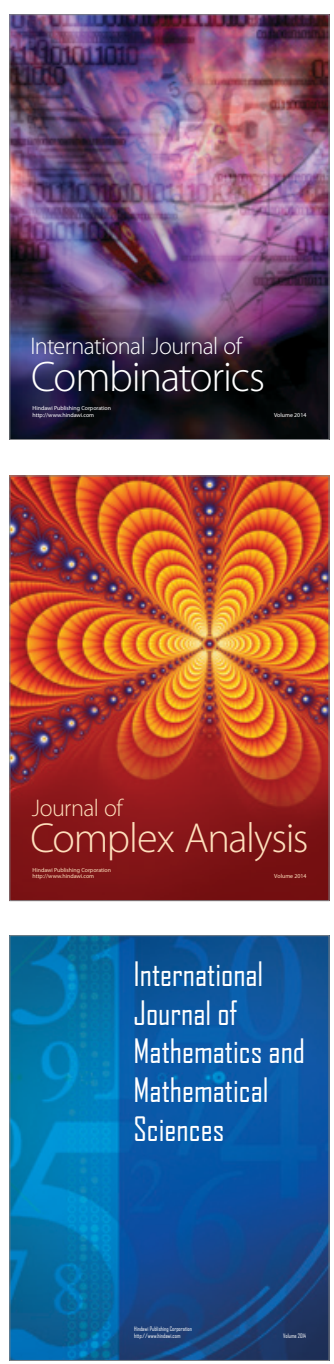
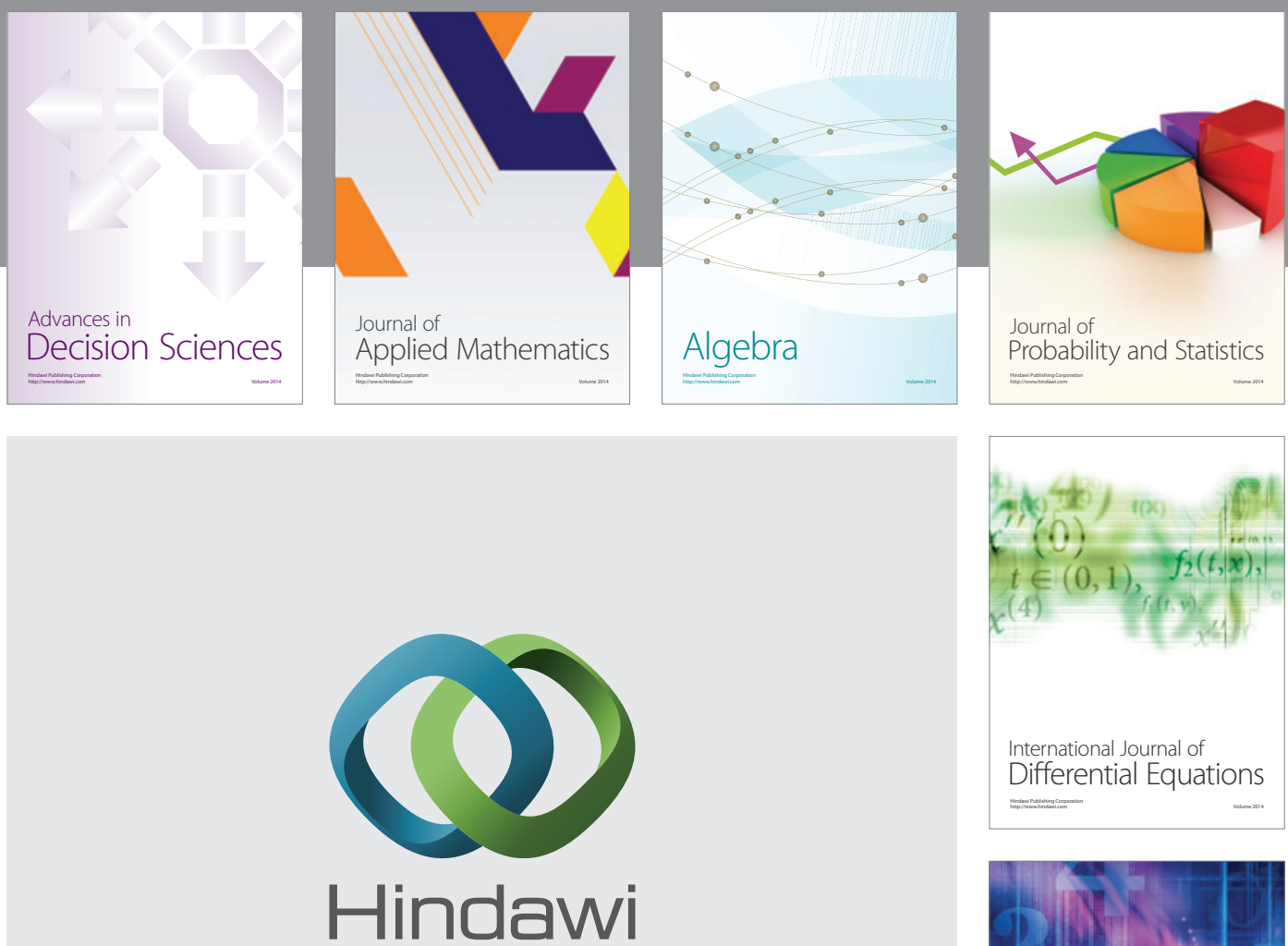

Submit your manuscripts at http://www.hindawi.com
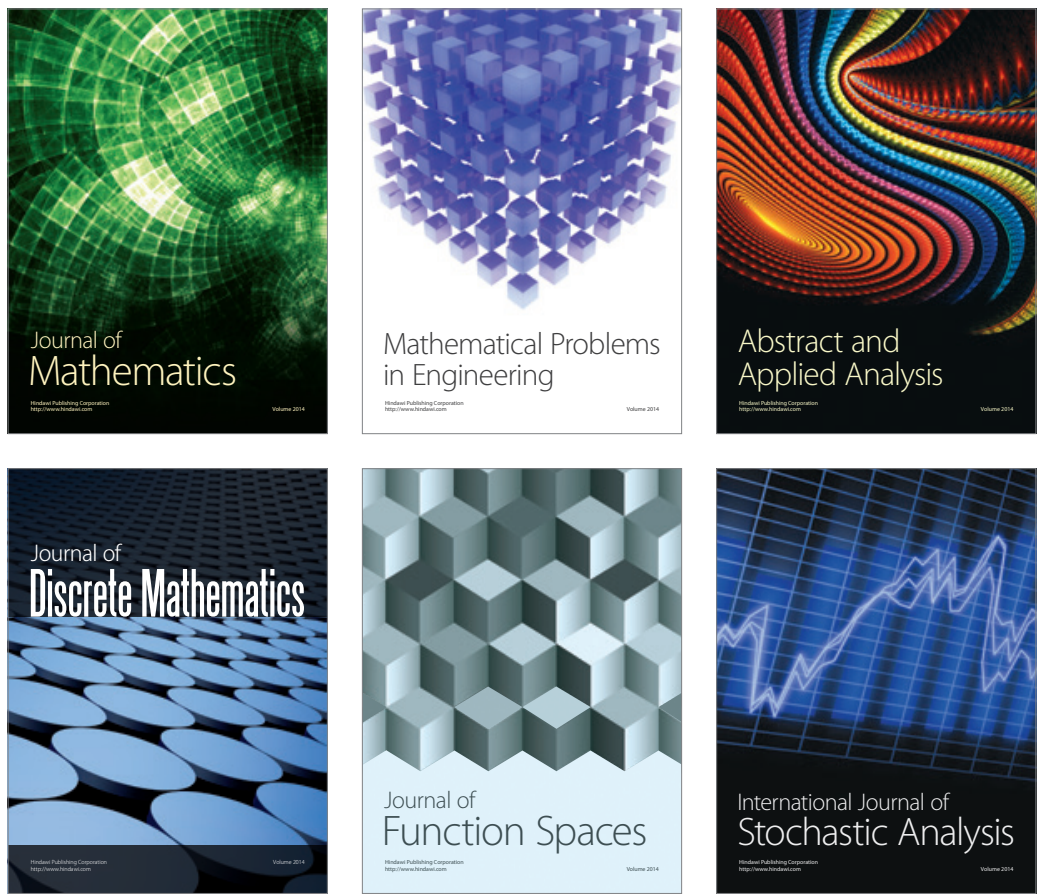

Journal of

Function Spaces

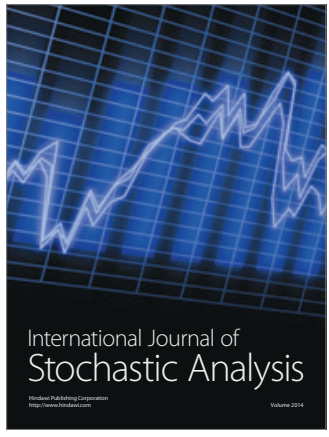

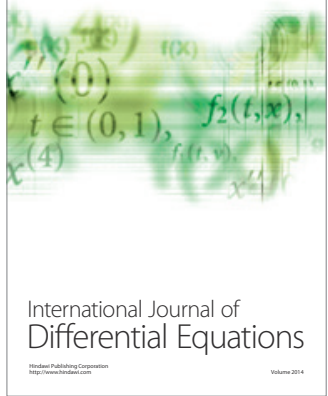
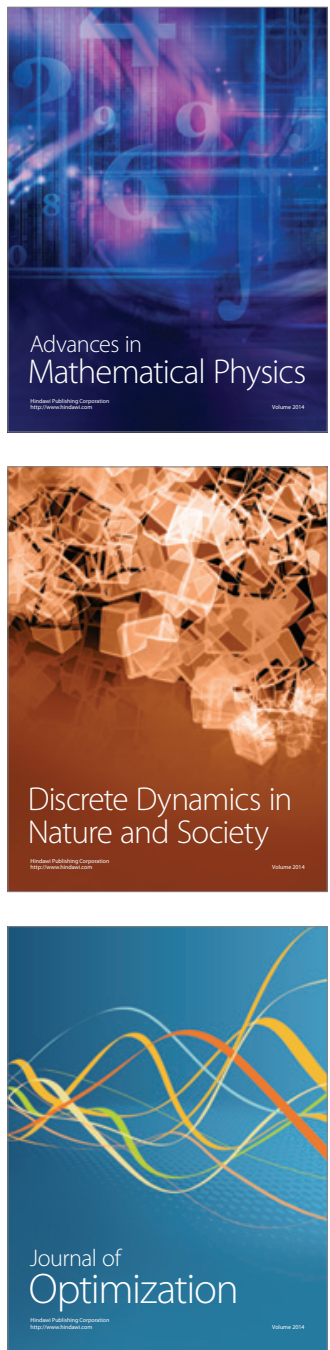